\title{
Thesis
}

\section{Contribution towards intelligent service management in wearable and ubiquitous devices}

\author{
Pedro Castillejo *, José-Fernán Martínez and Lourdes López \\ Next-Generation Networks and Services (GRYS) research group, Research Center on Software Technologies and \\ Multimedia Systems for Sustainability (CITSEM), Technical University of Madrid (UPM), Spain \\ E-mails: pedro.castillejo@upm.es, jf.martinez@upm.es,lourdes.lopez@upm.es
}

Keywords: Wireless Sensor Networks, wearable sensors, e-Health, smart spaces, security, privacy

On July 7th, 2015, Pedro Castillejo defended his Ph.D. thesis titled "Contribution towards intelligent service management in wearable and ubiquitous devices" at the Technical University of Madrid (UPM). This thesis has been developed within the European research project "LifeWear-Mobilized Lifestyle with Wearables" funded by the Spanish Ministry of Industry, Tourism and Trade in the framework of the European Research Program ITEA 2 (Information Technology for European Advancement 2). The thesis has been supervised by Prof. Martínez and Prof. López. The dissertation defense took place in a public, open presentation held at the School of Telecommunications Systems and Engineering of the Technical University of Madrid (ETSIST-UPM). The thesis committee included Dr. Juan Carlos Dueñas López, Dr. Martina Eckert, Dr. Luigi Glielmo, Dr. Juan Ramón Velasco Pérez and Dr. Carlos García Rubio. The thesis was read and approved by his thesis committee, receiving the highest rating (awarded with honours).

\section{Thesis summary}

The main objective of this thesis is defining and designing a solution for intelligent service management

*Corresponding author. E-mail: pedro.castillejo@upm.es. in wearable and ubiquitous devices so as to solve the heterogeneity issues that are presented when dealing with interoperability and interconnectivity of devices and software of different nature. Additionally, a security schema based on trust domains is proposed as a solution to the privacy problems arising when private data (e.g. biomedical parameters or user identification) is broadcasted in a wireless network.

Nowadays, society is shifting towards a growing interest and concern on health care. This phenomenon can be acknowledged by two facts: first, the increasing proportion of people practicing some kind of healthy activity (sports, balanced diet, etc.). Secondly, the growing number of commercial wearable smart devices (smartwatches or bands) able to measure physiological parameters such as heart rate, breathing rate, distance or consumed calories.

A large number of applications combining both facts are appearing. These applications are not only able to monitor the health status of the user, but also to provide recommendations about routines in order to improve the mentioned health status. In this context, wearable devices merged with the Internet of Things (IoT) paradigm enable the proliferation of new market segments for these health wearable-based applications, such as Ambient Assisted Living (AAL). These applications can provide solutions for the elderly or baby 


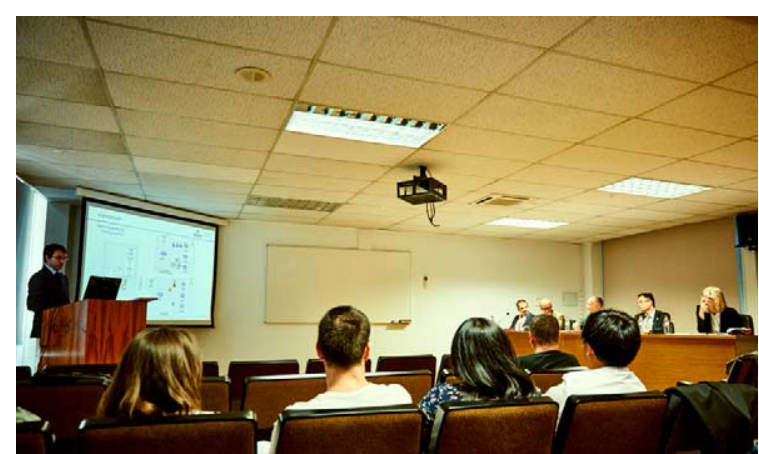

Fig. 1. Picture taken during the Ph.D. thesis defense.

care, in-hospital or in-home patient monitoring, security and defense fields or an unforeseen number of future applications.

The introduced IoT paradigm can be developed with the usage of existing Wireless Sensor Networks (WSNs) by connecting the novel wearable devices to them. In this way, the migration of new users and actors to the IoT environment will be eased. However, a major issue appears in this environment: heterogeneity. In fact, there is a large number of operating systems, hardware platforms, communication and application protocols or programming languages (each of them with unique features) that may present challenges when a holistic, seamless solution is being developed.

The contributions presented in this thesis have been made after a comprehensive state-of-the-art analysis, and include the design of a Wearable Device Service Bus (WDSB) integrating the technologies collected in the requirement analysis (ESB, WWBAN, WSN and IoT). Applications are able to access the WSN services regardless of the platform and operating system where they are running [1]. Besides, this pro- posal also includes the design of a Wearable InterDomain communication Protocols set (WIDP) which integrates lightweight protocols suitable to be used in low-capacities devices (REST, JSON, AMQP, CoAP, etc... ) [2]. Furthermore, a security solution for service management based on a trustworthy domains model to deploy security services in WSNs has been designed [3].

Although the proposal is a generic framework for applications based on services provided by wearable devices, a specific application scenario for testing purposes has been included. In this validation scenario (defined inside the European research project "LifeWear-Mobilized Lifestyle with Wearables") it has been presented an autonomous physical condition performance system, based on a WSN, bringing the possibility to include several elements in an IoT scenario: a smartwatch, a wireless physiological monitoring device and a smartphone.

\section{References}

[1] P. Castillejo, J.-F. Martínez Ortega, J. Rodriguez-Molina and A. Cuerva, Integration of wearable devices in a wireless sensor network for an E-health application, IEEE Wireless Communications 20(4) (2013), 38-49.

[2] P. Castillejo, J.-F. Martínez Ortega, L. López and G. Rubio, An Internet of Things approach for managing smart services provided by wearable devices, International Journal of Distributed Sensor Networks 2013 (2013), Article ID 190813, 9 pages. doi: $10.1155 / 2013 / 190813$.

[3] P. Castillejo, J.-F. Martinez Ortega, L. López and J. Antonio Sánchez Alcón, SensoTrust: Trustworthy domains in Wireless Sensor Networks, International Journal of Distributed Sensor Networks 2015 (2015), Article ID 484820. doi:10.1155/2015/484820. 